\title{
Basic FGF in Adult Rat Brain: Cellular Distribution and Response to Entorhinal Lesion and Fimbria-Fornix Transection
}

\author{
Fernando Gómez-Pinilla, Jason Won-Kyun Lee, and Carl W. Cotman \\ Department of Psychobiology, University of California, Irvine, California 92717
}

Basic fibroblast growth factor (bFGF) is a potent trophic factor for neurons and astrocytes and recently has been implicated in the pathology of Alzheimer's disease. In order to better understand the role of bFGF in normal brain function and during pathology, we have analyzed its anatomical distribution and its response to injury in the CNS. Double-staining immunohistochemistry showed that bFGF immunoreactivity was localized in astrocytes, in select neuronal populations, and occasionally in microglial cells throughout the normal rat brain. Neuronal populations that showed bFGF immunoreactivity included septohippocampal nucleus, cingulate cortex, subfield CA2 of the hippocampus, cerebellar Purkinje cells, cerebellar deep nuclei, facial nerve nucleus, and the motor and spinal subdivisions of the trigeminal nucleus and facial nerve nucleus. The pattern of bFGF immunoreactivity in the hippocampus was examined following entorhinal cortex lesion or fimbria-fornix transection. After entorhinal cortex lesion, bFGF immunoreactivity increased in the outer molecular layer of the dentate gyrus ipsilateral to the lesion. The lesion effect on bFGF immunoreactivity was expressed as an increase in the number of bFGF astrocytes, as an increase in the intensity of bFGF immunoreactivity within astrocytes, and as an increase of bFGF immunoreactivity in the surrounding extracellular matrix, relative to the contralateral side. The time course and pattern of reorganization paralleled the sprouting of septal cholinergic terminals in response to the same type of lesion, suggesting that bFGF may play an important role in lesion-induced plasticity. After transection of the fimbria-fornix, chronic infusion of bFGF appeared to preserve NGF receptors on neurons within the medial septal complex and, as previously reported, prevent the death of medial septal neurons. Therefore, it appears that bFGF infusion, which has been shown to increase the synthesis of NGF by astrocytes (Yoshida and Gage, 1991), also helps enable neurons to respond to NGF. This suggests that after injury bFGF may participate in a cascade of neurotrophic events, directly and indirectly facilitating neuronal repair and/or promoting neuronal survival.

Fibroblast growth factors (FGFs) are protein mitogens originally identified as promotors of fibroblast division in culture (Trowel and Willmer, 1939; Hoffman, 1940; see Walicke and Baird,

\footnotetext{
Received Apr. 11, 1991; revised Aug. 29, 1991; accepted Aug. 30, 1991.

We thank Jennifer Kahle and Linda Huba for their editorial assistance and comments in the preparation of the manuscript. This research was funded by Aging Program Project AGO0538, NIMH 19691 and HD 2436.

Correspondence should be addressed to Fernando Gómez-Pinilla, Ph.D., Department of Psychobiology, University of California, Irvine, CA 92717.

Copyright $\odot 1992$ Society for Neuroscience $0270-6474 / 92 / 120345-11 \$ 05.00 / 0$
}

1988, for review). FGF was recognized as an important neurotrophic factor after high levels of FGF mitogenic activity were detected in the brain (Gospodarowicz, 1974; Morrison and DeVellis, 1981). A large variety of cell types including astrocytes, capillary endothelial cells, vascular smooth muscle cells, and neurons (Baird and Walicke, 1989) have been shown to respond to both the acidic and basic forms of FGF. The basic form of FGF (bFGF) has been isolated from brain tissue (Gospodarowicz et al., 1984; Esch et al., 1985; Abraham et al., 1986), and bFGF receptors have been identified in brain (Inamura et al., 1988; Lee et al., 1989; Isacchi et al., 1990; Reid et al., 1990). In addition, bFGF has been considered a potent neurotrophic factor active at low concentrations as a promotor of neuronal survival and neurite extension in vitro (Walicke, 1988b; Grothe et al., 1989) and in vivo (Sievers et al., 1978; Anderson et al., 1988; Otto et al., 1989). bFGF has a broad spectrum of action and can be stored in many tissue compartments due to its high affinity for heparan sulfate proteoglycans present in the extracellular matrix (Gospodarowicz et al., 1984). Although much has been learned about the physiological properties of bFGF from in vitro studies, the exact cellular location and action of bFGF in the brain are still a matter of debate. Information on the locus of bFGF in the brain is essential to a better understanding of the functional role of bFGF.

Trophic factors may participate not only in the maintenance of the normal cytoarchitecture of the brain but also in its response to injury. When damaged, neurons appear to react in a manner dependent on the type of injury. For example, neurons that are axotomized generally dedifferentiate and degenerate, while the remaining healthy neurons may undergo a sprouting reaction. Neurotrophic factors may participate in this response to injury by triggering growth and preventing degeneration. Indeed, previous work has suggested that the sprouting reaction may involve the induction of select neurotrophic factors that increase after injury (Nieto-Sampedro et al., 1982). bFGF is a good candidate since it acts on a large variety of neurons and, as previous work suggests, bFGF immunoreactivity increases near the site of injury (Finklestein et al., 1988). At present, however, the response of bFGF in areas of denervation or axotomy has not yet been described.

Studies in Alzheimer's disease (AD) postmortem brain tissue have shown that axon sprouting occurs in the dentate gyrus, probably early in the disease (Geddes et al., 1985, 1986), and as the disease progresses, sprouting appears to become involved in senile plaque formation (Geddes et al., 1986). It has been hypothesized that plaque formation may involve a local increase in neurotrophic factors (Ramon y Cajal, 1928; Geddes et al., 1986), and recently bFGF has been identified in plaques (Gómez-Pinilla et al., 1990; Stopa et al., 1990). Imbalances in the 
levels of neurotrophic factors may contribute to neuronal health versus vulnerability in this disease. Studies in animal models may provide clues to understanding the regulation of bFGF in response to injury in normal brains and those of patients with AD.

The hippocampal formation provides a suitable model for detailed study of the response to injury and consequent anatomical reorganization because of its well-defined structure and its well-characterized axon sprouting (Cotman and Anderson, 1989). The dentate gyrus (DG) receives extrinsic afferents from the entorhinal cortex (EC), the septum, and a variety of other ascending and descending nuclei. Destruction of the entorhinal input evokes a powerful sprouting of cholinergic septal afferents as well as other inputs in the dentate molecular layer (Cotman et al., 1973) that may involve an increase in local bFGF activity. In this study, we have used a monoclonal antibody that specifically recognizes the conformation of the bFGF molecule associated with its biological activity (Matsuzaki et al., 1989). Such an antibody has not been previously applied to the study of the distribution and regulation of bFGF in brain. In this article, we examine the locus of "active" bFGF in the hippocampus and throughout the rodent brain. We have also studied the effects of EC lesions and fimbria-fornix (FF) transections on local changes in bFGF immunoreactivity.

\section{Material and Methods}

\section{Subjects and surgery}

Sprague-Dawley male rats 3-4 months old anesthetized with Nembutal $(50 \mathrm{mg} / \mathrm{kg}$, i.p.) received unilateral (right) transection of the fimbriafornix $(\mathrm{FF} ; n=20)$ by a knife cut or electrolytic lesion of the entorhinal cortex (EC; $n=20$ ), according to NIH guidelines. After surgery, experimental rats were allowed to survive for 2,7 , or $14 \mathrm{~d}$ and then processed for histology. Lesioned rats and intact control rats $(n=4)$ were deeply anesthetized with Nembutal $(75 \mathrm{mg} / \mathrm{kg}$, i.p.) and then killed by intracardial perfusion with $200 \mathrm{ml}$ of PBS, followed by $500 \mathrm{ml}$ of $4 \%$ paraformaldehyde in Sorensen buffer, $\mathrm{pH}$ 7.3. The brains were removed, postfixed for $15 \mathrm{~min}$ in fresh solution of the same fixative, and stored overnight in $20 \%$ sucrose. Serial coronal sections $(30 \mu \mathrm{m})$ between the levels of the medial septum (MS) and caudal hippocampus were cut in a cryostat and processed for histology as described below. Alternate sections were stained with cresyl violet or AChE histochemistry to visualize the general cytoarchitecture of the areas under study. The quality of the EC lesion or FF transection was verified by corroborating the typical pattern of cholinergic fibers obtained with AChE histochemistry in the hippocampus (Cotman et al., 1973). Chronic infusion of bFGF in FF-lesioned rats was performed in a separate set of experiments as described below.

\section{Immunohistochemistry}

The tissue sections were first treated with $1 \% \mathrm{H}_{2} \mathrm{O}_{2}$ in cold $100 \%$ methanol to inactivate the endogenous peroxidase activity. Alternate sections were then incubated at $4^{\circ} \mathrm{C}$ for $24 \mathrm{hr}$ in anti-NGF receptor antibody
(NGFR; mouse monoclonal 192, kindly provided by Dr. E. Johnson, Jr., Washington University) at a concentration of $5 \mu \mathrm{g} / \mathrm{ml}$ in PBS, $\mathrm{pH}$ 7.2 , containing $2 \% \mathrm{BSA}$; or for $24 \mathrm{hr}$ in mouse monoclonal anti-bFGF antibody (UBI Technology, NY) at a concentration of $1.8 \mu \mathrm{g} / \mathrm{ml}$; or for $24 \mathrm{hr}$ in rabbit monoclonal anti-glial fibrillary acidic protein antibody (GFAP; Dakopatts Labs, Glostrup, Denmark) at a concentration of 29 $\mu \mathrm{g} / \mathrm{ml}$, diluted in PBS containing $0.1 \%$ Triton X-100 and $2 \%$ BSA. The sections were then rinsed three times over $30 \mathrm{~min}$ and incubated in ratadsorbed biotinylated horse anti-mouse IgG (for NGFR or bFGF immunostaining) or biotinylated goat anti-rabbit IgG (for GFAP staining), for $1 \mathrm{hr}$. After several rinses, sections were incubated in avidin-HRP complex for $1 \mathrm{hr}$, rinsed, incubated in 3,3'-diaminobenzidine (DAB; $0.5 \mathrm{mg} / \mathrm{ml}$ in Tris buffer, $10 \mathrm{~min}$ ), and then reacted for $10 \mathrm{~min}$ with fresh DAB solution containing $0.01 \% \mathrm{H}_{2} \mathrm{O}_{2}$.

\section{Double-staining immunohistochemistry}

In order to identify the cellular localization of bFGF, the same bFGFstained sections were costained with separate antibodies. An anti-GFAP antibody was used to detect astrocytes, and an anti-leukocyte common antigen (LCA; Dakopatts Labs) antibody was used to detect microglia. The color reaction to detect the first antibody (anti-bFGH) was developed with nickel chloride combined with $D A B$ to yield a blue-black reaction product instead of the typical brown reaction product. Sections were thoroughly rinsed in PBS, reacted for $15 \mathrm{~min}$ in excess of avidin, and then reacted for another $15 \mathrm{~min}$ in excess of biotin. After rinsing in PBS, sections were incubated overnight in anti-GFAP antibody or anti-LCA antibody. The rest of the process was exactly the same as described above, except that the second antibody was detected with $\mathrm{DAB}$ only to yield a brown reaction product. All the sections were thoroughly rinsed with PBS, mounted and dehydrated with increasing gradients of ethanols, and then coverslipped with Depex mounting media (BDH Ltd., Poole, England). Sections were examined on an Olympus BH-2 microscope. Controls for antibody specificity did not contain primary antibody, or the primary antibody was replaced by another antibody of common use in our laboratory.

\section{$b F G F$ chronic ventricular infusion after fimbria-fornix transection: cannula implantation}

These experiments were designed to study the effect of chronic infusion of bFGF to rescue neurons that possess NGFR in the medial-septalvertical diagonal band (MS-vDB) complex. Two hours before the lesion and under deep Nembutal anesthesia $(50 \mathrm{mg} / \mathrm{kg}$, i.p.), a cannula $(25$ gauge, stainless steel) connected to an osmotic minipump (model 2002, Alza, Palo Alto, CA; flow rate, $0.5 \mu \mathrm{l} / \mathrm{hr}$ for $14 \mathrm{~d}$ ) was implanted into the right lateral ventricle. In experimental rats $(n=10)$, the minipump delivered bFGF diluted to $500 \mathrm{ng} / \mathrm{ml}$ in sterile saline containing $0.1 \%$ $\mathrm{v} / \mathrm{v}$ autologous rat serum as a carrier. Control rats received sterile saline $(n=3)$ with $0.1 \%$ autologous rat serum or no infusion $(n=3)$. Fourteen days after surgery all rats were killed before previous treatment with diisopropylfluorophosphate (Sigma; $2 \mathrm{mg} / \mathrm{kg}$ in saline) to inhibit new synthesis of AChE.

\section{Quantitative analysis}

Lesion effects on the number of astrocytes in the hippocampus. To assess the effects of EC lesion or FF transection, all bFGF cells contained within an area of $100 \mu \mathrm{m}^{2}$ in the outer molecular layer and lacunosum moleculare of the dentate gyrus (DG), and in the stratum oriens of the

\footnotetext{
Figure 1. Double-staining immunohistochemistry illustrating the locus of bFGF in the rat brain. bFGF was stained to develop a blue-black reaction product using the DAB-NiCl method. $A$ shows the typical pattern of bFGF immunoreactivity in which mostly blue-black cell bodies were observed. $B$ shows the presence of bFGF in astrocytes as was demonstrated by coimmunostaining sections with the astrocyte marker GFAP developed with DAB, so that the astrocytes stained with a brown reaction product. This costaining showed strong immunoreactivity for bFGF (blue-black) within the nucleus and the perinuclear area of astrocytes. $C$ demonstrates bFGF in microglia by coimmunolabeling sections with the macrophage marker LCA (blue-black) and with the bFGF (brown). Scattered microglial profiles showed bFGF (arrow) in soma. $D$ shows the presence of bFGF in cell body and proximal processes of reactive astrocytes nearby the injury site of an FF lesion involving the cerebral cortex. The photograph was taken from a coronal section of the cerebral cortex double-stained using anti-bFGF and anti-GFAP antibodies. $E$ shows typical appearance of GFAP-labeled astrocytes in a control section. $F$, Double-staining immunohistochemistry using anti-bFGF and anti-GFAP antibodies to show typical bFGF immunoreactivity associated with blood vessel elements. Astrocytes $(E$, open arrow) surrounding the blood vessel and perivascular endothelial cells ( $E$, solid arrow) exhibited positive bFGF immunoreactivity. Photographs $A-C, E$, and $F$ were obtained from coronal sections of the hippocampus. Scale bar $(E), 10 \mu \mathrm{m}$ for $A-F$.
} 


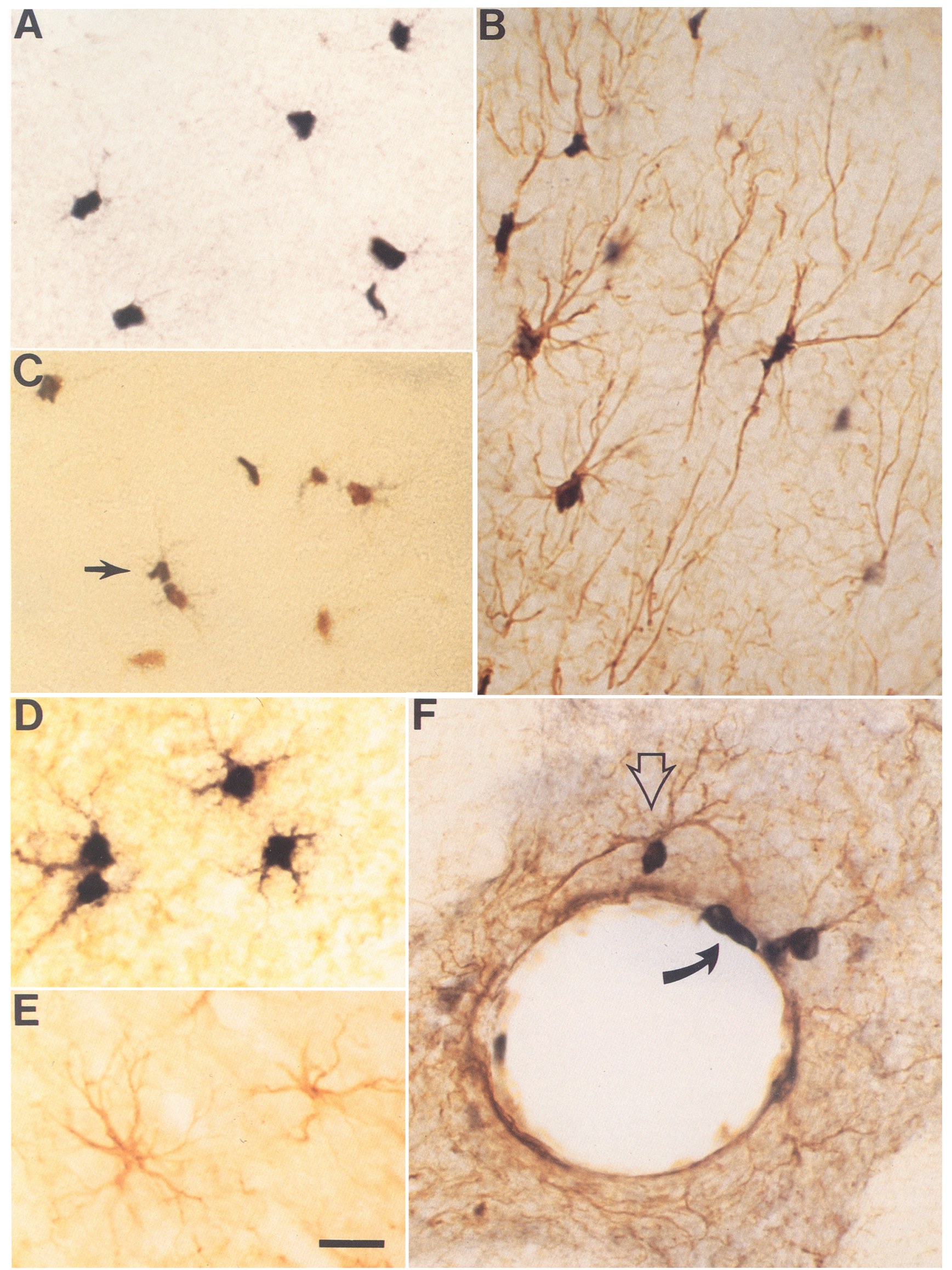






Figure 2. Typical pattern of bFGF immunoreactivity in the hippocampus. A, Coronal section showing bFGF-positive astrocytes are widely distributed in the hippocampus while neuronal bFGF is restricted to the CA2 region (box). B shows higher magnification of the boxed CA2 region from $A$. bFGF was concentrated in the nucleus and within proximal dendritic processes of pyramidal neurons. CAl (cal) and CA3 (ca3) regions of the hippocampus are labeled as a reference; $I m$, lacunosum moleculare; $h$, hilus of the DG; $g$, granular layer of the DG. Scale bars, $100 \mu$ m.

CA3 region of the hippocampus, were counted bilaterally. These regions were selected according to previous studies (Rose et al., 1976; Gage et al., 1988) indicating that astrocyte populations are susceptible to change after similar types of lesions. bFGF-immunoreactive cells were counted under camera lucida microscopy at $160 \times$ magnification, at five coronal planes separated by about $100 \mu \mathrm{m}$ from each other. The mean number of bFGF cells was calculated for each individual DG for both the ipsilateral and contralateral sides of each animal. To minimize variations between animals, the ratio between the ipsilateral and contralateral sides for each rat was compared with each other using Student's $t$ test for independent group analysis.
Lesion effect on relative levels of $b F G F$ within astrocytes and extracellular matrix. We used computer image analysis procedures, as described by Smolen (1990), to assess possible lesion-induced changes in bFGF immunoreactivity in individual cells within the DG outer molecular layer. Slides were analyzed by computer-assisted optic densitometry with an MCID image processing system (Imaging Inc., St. Catherines, Ontario, Canada). bFGF optic density was measured in the DG outer molecular layer ipsilaterally and contralaterally, at three coronal planes separated by about $100 \mu \mathrm{m}$ from each other. Three slide sections were analyzed per animal. Optical density readings were taken from all the cells that expressed bFGF immunoreactivity within each of the five 

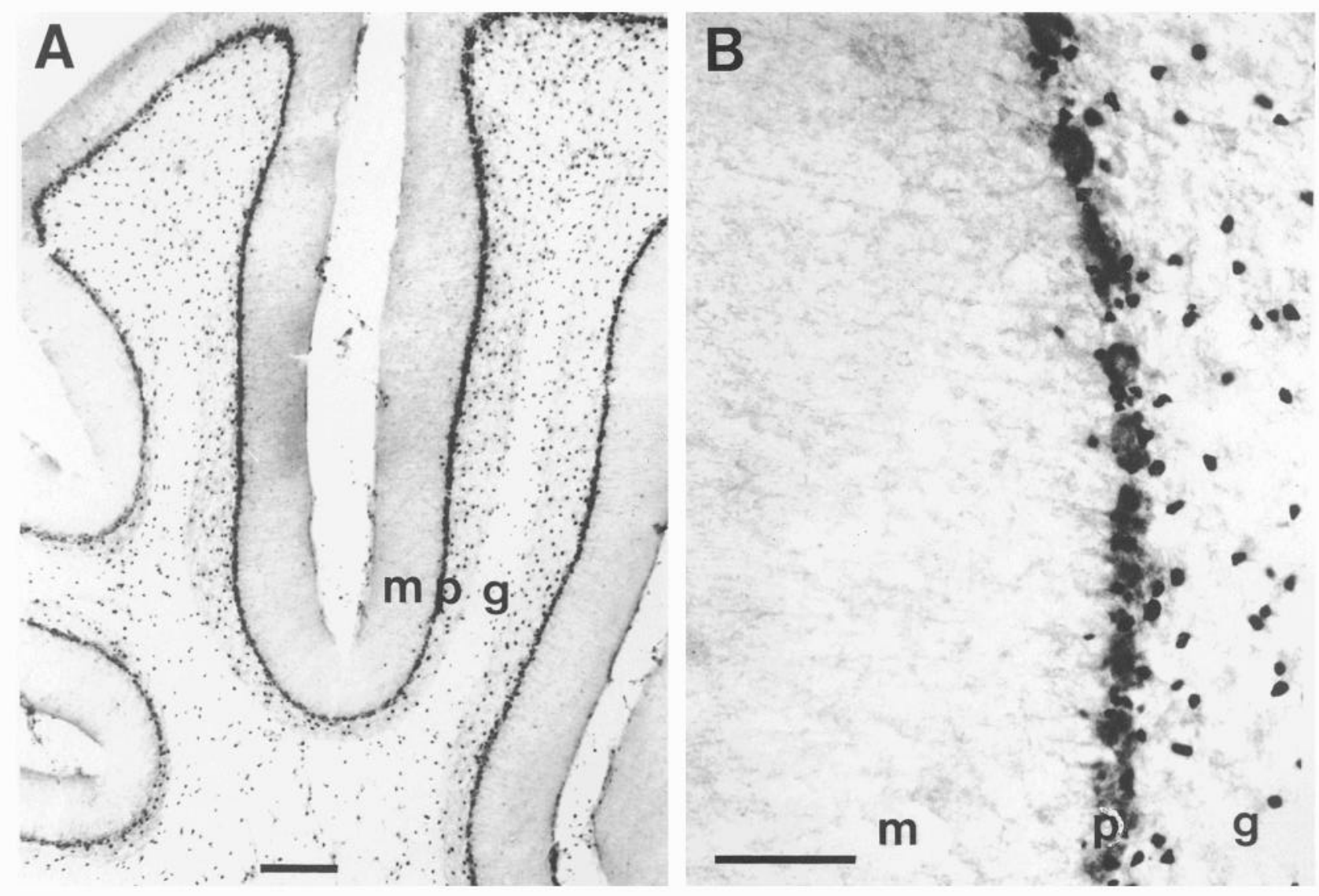

Figure 3. Typical pattern of bFGF immunoreactivity in the cerebellum. $A$, Sagittal section of a cerebellar lobule showing bFGF immunoreactivity in the Purkinje cell layer $(p)$ and in the granular cell layer $(g)$. B. Enlargement of the cerebellar cortex showing that bFGF immunoreactivity is localized in cell bodies of Purkinje cells and in astrocytic elements within the granular layer. There is weak or nondetectable bFGF immunoreactivity in the molecular layer $(m)$. Scale bars: $A, 300 \mu \mathrm{m} ; B, 100 \mu \mathrm{m}$.

computer fields, covering the complete medial-lateral extent of the DG outer molecular layer. Optical density readings were also taken from the surrounding extracellular matrix in a similar fashion. The means from the five computer fields were averaged for each individual DG, ipsilaterally and contralaterally. To minimize variations between animals, the mean ratio between ipsilateral and contralateral sides for each rat was calculated. This ratio was compared with normal littermate controls using a Student's $t$ test for independent groups analysis.

Effect of $b F G F$ ventricular infusion on NGFR-containing neurons. To assess the effect of chronic infusion of bFGF on axotomized septal neurons, all the NGFR-immunoreactive neurons were counted in the MS and vDB from each section. NGFR-immunoreactive cells were analyzed under camera lucida microscopy at $160 \times$ magnification, at five coronal planes separated by about $100 \mu \mathrm{m}$. The limit between MS and vDB was determined by using the anterior commissure as a reference (Gage et al., 1986). Only neurons that did not show any evidence of artifactual damage were considered. The results of neuronal survival were plotted as percentage of NGFR-containing neurons of the MS and $\mathrm{vDB}$ ipsilateral to the lesion relative to the contralateral hemisphere in both bFGF-treated and untreated control rats. The ratio of the mean number of neurons (ipsi/contra) stained with anti-NGFR antibody between control and experimental groups was compared using Student's $t$ test for independent groups analysis.

\section{Results}

\section{Distribution of bFGF in normal rat brain}

The main locus of bFGF immunoreactivity appeared to be in small cell bodies that resembled astrocytes (Fig. 1A). Doublestaining immunohistochemistry with anti-GFAP and anti-bFGF antibodies clearly identified these cells as astrocytes (Fig. $1 B$ ). This costaining also showed that the locus of bFGF in astrocytes appeared to be within the nuclear and perinuclear region. A few bFGF-immunostained cells that did not costain with anti-GFAP antibody showed positive immunoreactivity for anti-LCA antibody (Fig. 1C), a common marker for macrophage-line cells, which include microglia (McGeer et al., 1989). Other cells such as periventricular cells and perivascular endothelial cells (Fig. $1 E$ ) also showed bFGF immunoreactivity. Select neuronal elements within particular brain areas showed strong bFGF immunoreactivity. Neurons of CA2 subfield of the hippocampal formation stained throughout the rostrocaudal extent of the hippocampus (Fig. 2); CA3 subfield neurons stained only in the rostral hippocampus. Neurons in CA2 and CA3 showed strong bFGF immunoreactivity around the perinuclear area and weak staining in the cytoplasm and processes proximal to the soma. bFGF-positive neurons were also visualized in the septohippocampal nucleus, mainly in horizontal brain sections. Cingulate cortex neurons stained strongly throughout most of their rostrocaudal extents. In the cerebellum, somas of Purkinje cells and astrocytes within the granular layer showed bFGF immunoreactivity (Fig. 3). Deep nuclei of the cerebellum also showed bFGF immunoreactivity. In the brainstem, there was strong bFGF immunoreactivity within the facial nerve nucleus and the motor and spinal components of the trigeminal nucleus. 

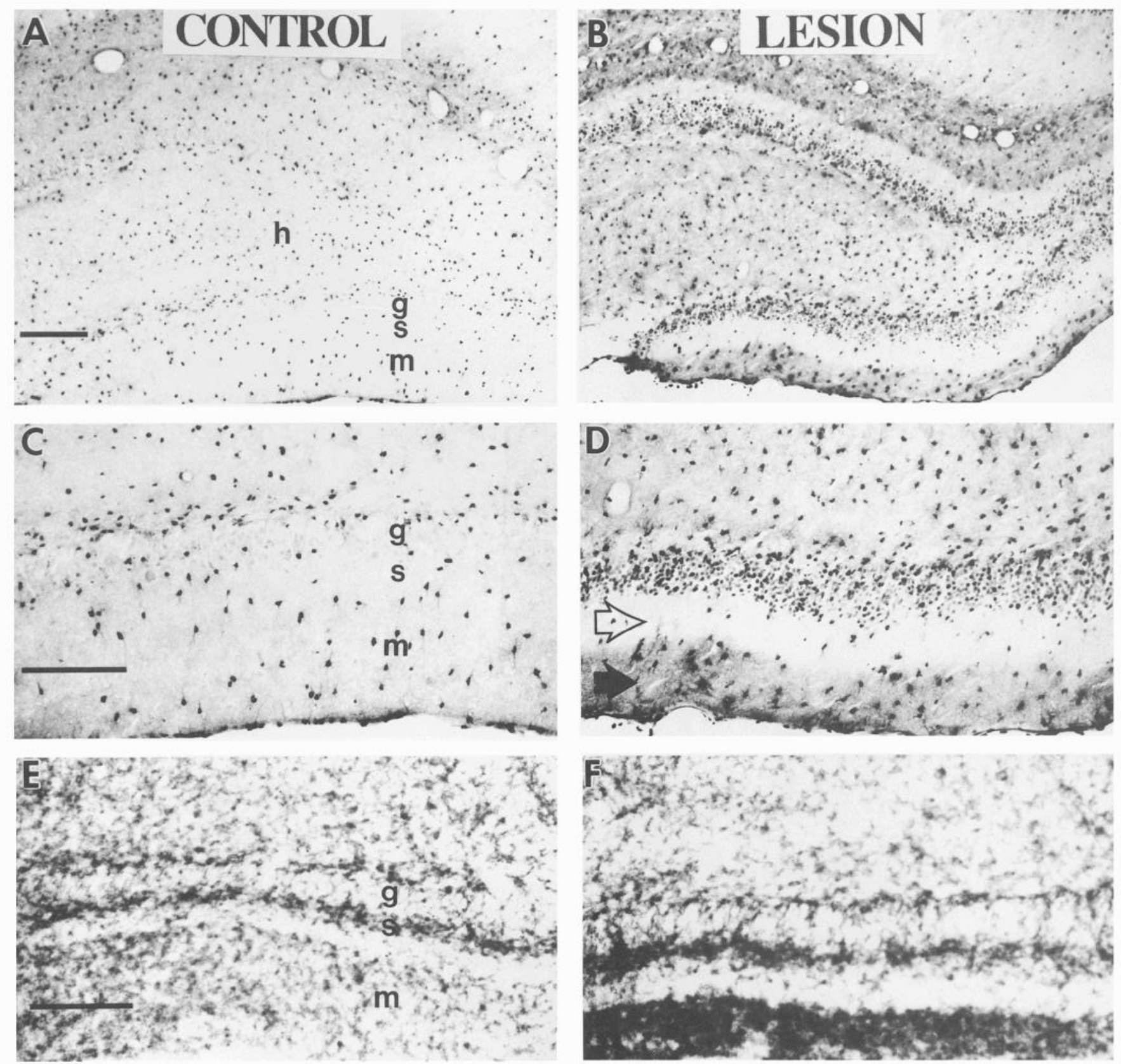

Figure 4. Effects of unilateral EC lesions on the pattern of bFGF immunoreactivity in the hippocampus. The outer molecular layer at the lesion side $(B)$ shows an increase in bFGF immunoreactivity as compared to the control side $(A)$. Enlargements of regions of the DG on the control side $(C)$ or lesion side $(D)$ show that on the lesion side there is an increase in bFGF immunoreactivity mainly in the outer molecular layer $(D$, solid arrow), while a clear zone appears in the middle molecular layer $(D$, open arrow). AChE histochemistry on the control side $(E)$ versus the lesion side $(F)$ shows that the increase of bFGF immunoreactivity displays a similar pattern to sprouting $(F)$ of cholinergic fibers in the DG outer molecular layer. The photograph was taken from a rat killed $2 \mathrm{~d}$ after EC lesion. $g$, granular cell layer of the DG; $h$, hilus of the DG; $s$, supragranular layer of the DG; $m$, molecular layer of the DG. Scale bars, $200 \mu \mathrm{m}$.

\section{Response of bFGF cells to lesion}

Response of bFGF cells to EC lesion. Particularly after an EC lesion, the hippocampus ipsilateral to a lesion showed an enhancement of bFGF immunoreactivity in the DG outer molecular layer (Fig. $4 B, D$ ). The lesion effects on bFGF immunoreactivity were expressed as a relative increase in the number of bFGF astrocytes, an increase in relative intensity of the bFGF immunoreactivity within astrocytes, and a relative increase of the bFGF immunoreactivity in the surrounding extracellular matrix as compared to the contralateral side. The increase in the number of astrocytes was already evident at postlesion day 2 , reached a maximum by day $7(p<0.01)$, and decreased to about normal levels by day 14 (Fig. 5). Computer densitometry showed an increase in bFGF immunoreactivity within individual astrocytes in the DG outer molecular layer reaching significance by postlesion day $7(p<0.05)$ and remaining through postlesion day $14(p<0.01)$, relative to the contralateral side and untreated controls (Fig. 6). The extracellular matrix surrounding bFGF-positive astrocytes also showed an increase in 


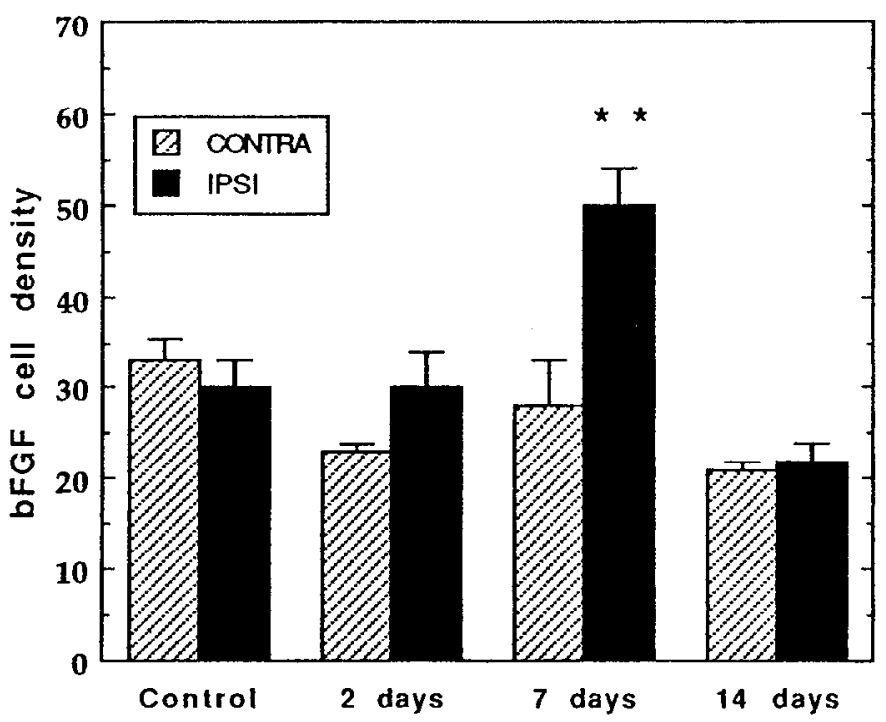

Figure 5. Graph illustrating the effects of EC lesions on the number of bFGF astrocytes in the DG outer molecular layer. The graph shows the average ratio of bFGF-immunoreactive astrocytes observed under camera lucida on the ipsilateral side versus the contralateral side. On the ipsilateral side, the number bFGF cells is increased by $2 \mathrm{~d}$ after EC lesion, reaches significance by postlesion day $7(p<0.01)$, and returns to about normal levels by postlesion day 14 . The data are expressed as mean number of cells per $100 \mu \mathrm{m}^{2}$ of tissue sample. Error bars are SEM (**, $p<0.01$; Student's $t$ test).

bFGF immunoreactivity with a similar time course (Fig. 6). The bFGF immunostaining tended to increase in pyramidal cell layer of the CA2 region ipsilateral to the lesion, but this did not reach significance. Parallel serial sections stained for AChE histochemistry, a marker of axon sprouting in the hippocampus (Cotman et al., 1973), also showed an enhancement of the staining in the DG outer molecular layer. This intensification had a time course similar to that observed with anti-bFGF antibody (Fig. 4D,F).

Response of bFGF cells to FF lesion. After transection, there was a transient increase of bFGF immunoreactivity around the area of the injury starting at postlesion day 2 . Astrocytes showed increased bFGF immunoreactivity in their processes, and their cell bodies appeared to be enlarged and stained darker as compared to bFGF-immunoreactive astrocytes of normal rats (Fig. $1 D$ ). There was not any obvious change in bFGF immunoreactivity in either of the two hippocampi.

\section{Effects of bFGF infusion on axotomized NGFR-containing neurons}

Previous evidence has shown that FF transection causes degeneration and dedifferentiation of axotomized cholinergic neurons in the MS and vDB nuclei (Daitz and Powell, 1954; Gage et al., 1986; Sofroniew et al., 1987). NGF (Kromer, 1987; Montero and Hefti, 1988), as well as bFGF (Anderson et al., 1988; Otto et al., 1989), appears to be capable of reversing the effects of insult, NGF being slightly more effective. Since bFGF is able to stimulate astrocytes in culture to synthesize and secrete NGF (Yoshida and Gage, 1991), it might be expected that bFGF may also help maintain phenotypic properties such as NGF receptors in order that these neurons may be responsive to NGF. Thereby, NGF, which may increase in response to bFGF acting on glial cells, would have receptors on which to exert its effects. Ac-

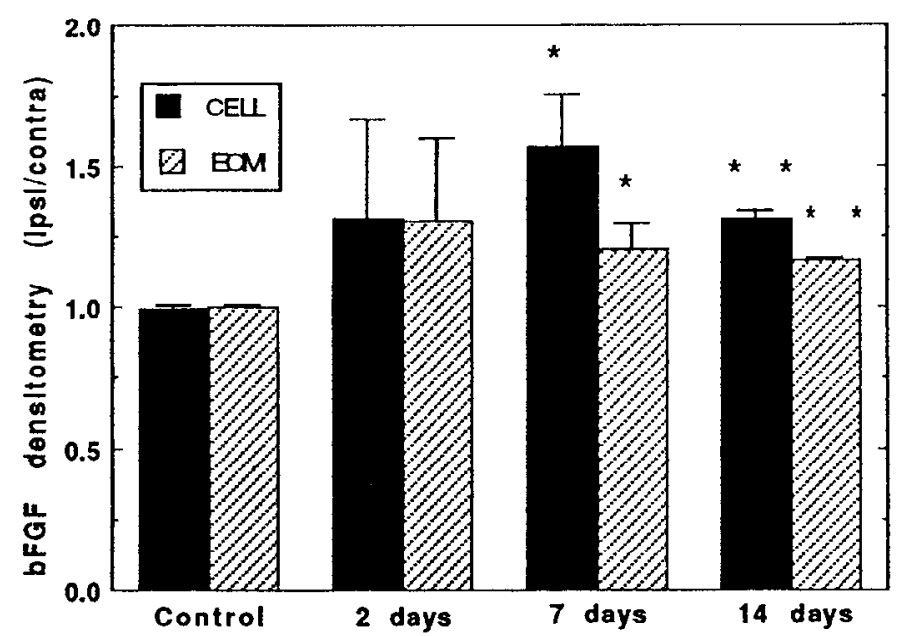

Figure 6. Graph illustrating the effects of EC lesions on the relative intensity of bFGF immunoreactivity within the DG outer molecular layer. Computer densitometry readings were taken from individual astrocytes and the surrounding extracellular matrix (ECM) from the immunohistochemical material. The graph shows the ratio of optical density on the ipsilateral (lesioned) side versus the contralateral (control) side. There is a relative increase in bFGF immunoreactivity as compared to intact controls in both astrocytes and extracellular matrix in the DG ipsilateral to the lesion, reaching significance by postlesion day $7(p<$ $0.05)$ and remaining through day $14(p<0.01)$. Error bars are SEM (*, $p<0.05$; ${ }^{* *}, p<0.01$; Student's $t$ test).

cordingly, we conducted experiments to study the possibility that chronic infusion of bFGF preserves neuronal NGFRs in the MS-vDB complex, thereby rescuing these neurons from certain death.

NGFR immunoreactivity in normal rats. Neurons of the MS and vDB showed strong NGFR immunoreactivity in the cell body and proximal sections of dendritic processes as described in detail elsewhere (Gómez-Pinilla et al., 1987). Neurons within the DB showed strong NGFR immunoreactivity in the cell bodies and in distal portions of their dendritic arbor (Fig. 7A,D).

NGFR immunoreactivity in lesioned rats. Two days after FF transection, there was a progressive decrease in the number of NGFR-immunoreactive neurons within the MS-vDB complex on the side ipsilateral to the lesion. These neurons lost intensity in NGFR immunoreactivity and developed clear signs of degeneration (Fig. $7 B, E$ ). At $14 \mathrm{~d}$ postlesion, the percentage of neurons surviving compared to the nonlesioned side was $40 \%$ for the MS and 39\% for the vDB (Fig. 8).

Effects of bFGF chronic treatment on lesioned rats. bFGF infused over the first 2 weeks after FF transection maintained NGFR density and ameliorated the apparent death of neurons that express NGFRs. The density of NGFR-immunoreactive neurons and intensity of their staining (Fig. 7C,E) were similar in both hemispheres in both the MS and vDB, from postlesion day 2 through postlesion day 14. Quantitation $14 \mathrm{~d}$ postlesion showed that bFGF-treated rats had a significant increase in survival of NGFR-immunoreactive neurons, from $40 \%$ to $63 \%$, in the MS $(p<0.05)$ and a significant increase from $39 \%$ to $82 \%$ in the $\mathrm{vDB}(p<0.01)$, relative to lesioned controls that did not receive treatment (Fig. 8). Alternate sections stained for $\mathrm{AChE}$ or with cresyl violet showed a similar preservation of the neuronal morphology to that shown with NGFR immunohistochemistry, in agreement with previous data (Anderson et al., 1987). 


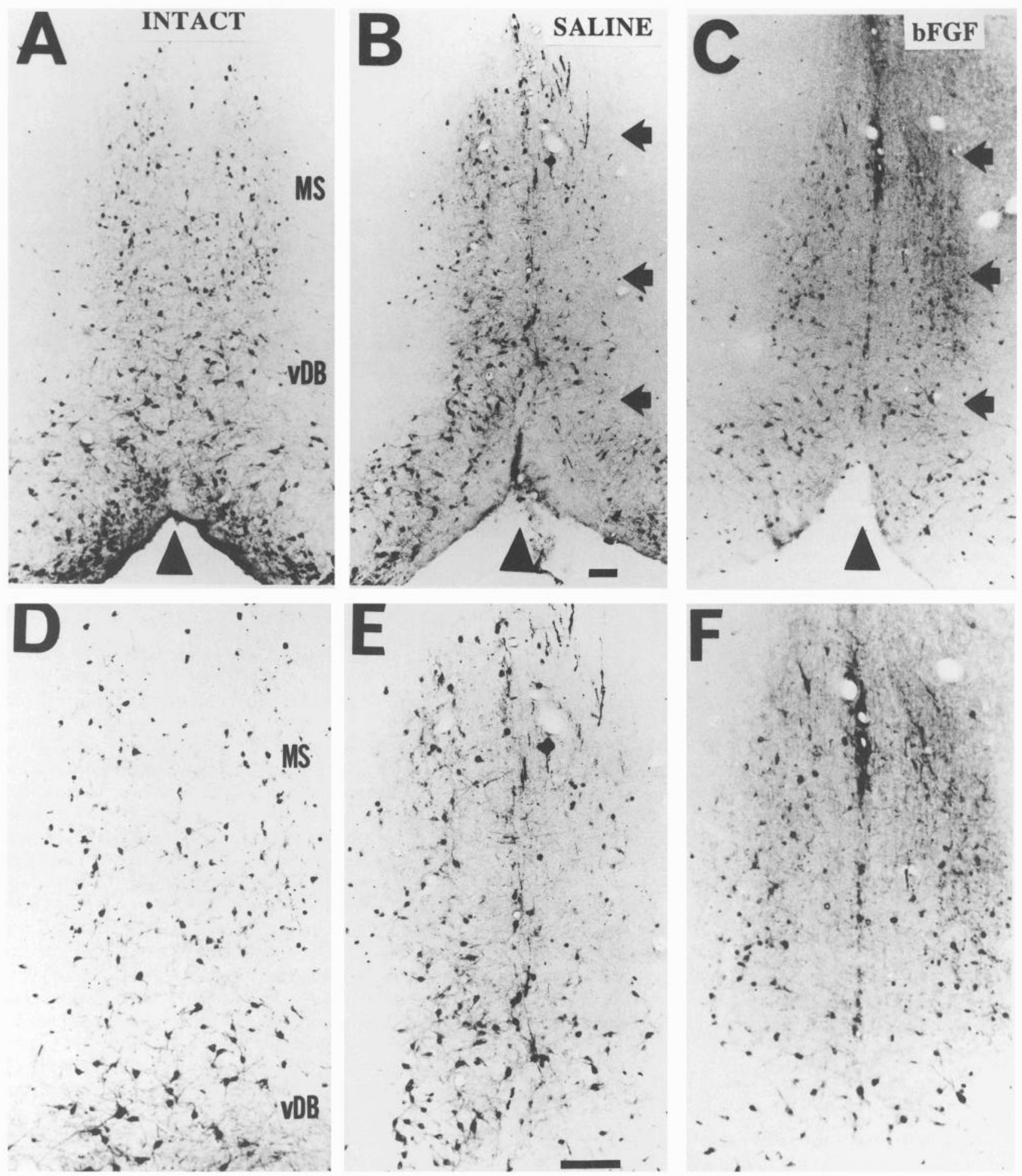

Figure 7. NGFR immunostaining at the level of the MS nucleus and vDB. The photographs at the bottom are enlargements of the MS-vDB region. $A$ and $D$ are representative samples from an intact rat showing the normal distribution of NGFR immunoreactivity. $B$ and $E$ are representative samples from a rat with FF transection that received intraventricular infusion of saline only. There was a loss of NGFR immunoreactivity in the lesion side $(B$, solid arrows) and the MS-vDB complex. $C$ and $F$ show the MS-vDB from a rat with FF transection that received chronic infusion of bFGF for 2 weeks. The bFGF-treated rats displayed a pattern in neuronal density and intensity of the NGFR immunostaining that was similar in the MS-vDB complex on both the lesioned $(C$, solid arrows) and unlesioned side. The midline in $A-D$ is indicated by arrowheads. Scale bars $(B$ for $A-C, E$ for $D-F), 100 \mu \mathrm{m}$. 


\section{Discussion}

Our results show that bFGF is located in astrocytes and select neuronal populations in the rat brain. The astrocytic location of bFGF indicates that it may have a potential trophic role in brain reactions that involve reactive astrocytosis. Thus, insofar as reactive astrocytosis appears to be a common brain response to many types of insults such as trauma or stroke, and even under pathological conditions such as AD (Vijayan et al., 1991), bFGF may be fundamental to brain function after injury. In this manner, astrocytes may provide trophic support to neurons and participate in injury-induced plasticity. While immunohistochemistry does not allow the detection of the locus of synthesis of bFGF, previous studies using other methodologies support the notion that the distribution of bFGF shown in our study corresponds to the loci of synthesis of bFGF in the brain. Studies in vitro clearly show that astrocytes can synthesize bFGF (Ferrara et al., 1988; Hatten et al., 1988). Emoto et al. (1989) have used in situ hybridization to identify the loci of bFGF mRNA in the rat brain, though they were unable to detect an astrocytic locus for bFGF mRNA. They did detect bFGF mRNA in astrocytes in culture but only when they were organized in clusters. They suggest that in situ hybridization is unable to resolve the low levels of bFGF mRNA present in isolated cells such as astrocytes. Their observations did, however, note the presence of bFGF mRNA in clusters of neurons in the same regions described by us such as $\mathrm{CA} 2$ hippocampal region and cingulate cortex. It is unlikely that astrocytes accumulate bFGF since there are no nearby sources and since the distribution of astroglial bFGF is similar throughout the brain even near bFGFpositive neurons.

Our results on the locus of bFGF in the brain are partially in disagreement with previous studies by Pettmann et al. (1986). According to these authors, bFGF is localized exclusively in neurons in the normal brain. In our study, however, only a restricted number of neuronal populations showed bFGF immunoreactivity in the adult rat brain. Possible sources of disagreement between our studies and their studies may be due to differences in the antibodies used and/or differences in the age of the animals. Our studies made use of a well-characterized monoclonal antibody that recognizes the molecular conformation of bFGF associated with its biological activity (Matsuzaki et al., 1989). Furthermore, the studies of Pettmann et al. (1986) were performed in neonatal rats and described a decrease of bFGF immunoreactivity in neurons with aging. Strong bFGF immunoreactivity was also observed in capillary endothelial cells, in general agreement with the recognized role of bFGF as a potent angiogenic factor (Folkman and Klagsbrun, 1987; Schweigerer et al., 1987). It has been previously shown that the anti-bFGF antibody used in our study is able to block angiogenesis in vitro (Matsuzaki et al., 1989). Thus, the presence of "active" bFGF in brain capillaries may suggest a physiological role of bFGF in revascularization following brain injury.

The widespread distribution of brain astrocytes displaying bFGF immunoreactivity is significant in relationship to studies showing that a large variety of neuronal types are responsive to the action of bFGF. For example, in vitro studies (Hatten et al., 1988; Walicke, 1988a; Grothe et al., 1989) have shown that both acidic and basic FGF support the survival of neurons from a large variety of origins, including neocortex, hippocampus, entorhinal cortex, striatum, septum, and thalamus. The fact that not all of these neuronal types are responsive to the action of

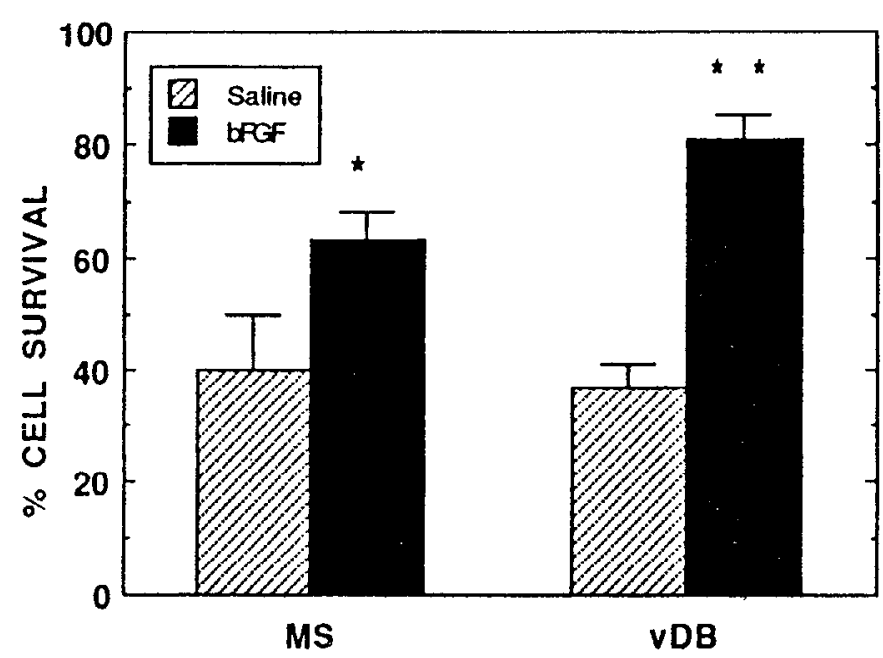

Figure 8. Graph illustrating the effects of bFGF on the survival of NGFR-immunoreactive neurons 2 weeks after FF transection. The saline-treated rats show only a $40 \%$ survival of NGFR neurons in the MS-vDB nuclei ipsilateral to the lesion. In contrast, the group of rats that received chronic infusion of bFGF for 2 weeks following the lesion show a $63 \%$ NGFR neuronal survival $(p<0.05)$ in the MS and an $82 \%$ NGFR neuronal survival $(p<0.01)$ in the vDB. Error bars are SEM $\left({ }^{*}, p<0.05 ;{ }^{* *}, p<0.01 ;\right.$ Student's $t$ test $)$.

NGF (Walicke and Baird, 1988) illustrates the broader, more pervasive role of $b F G F$ as compared to NGF.

In the present study, EC lesion increased bFGF immunoreactivity in the DG. It has been previously shown that destruction of entorhinal input in rats (Cotman et al., 1973) or entorhinal cell death in humans, for example, in AD (Geddes et al., 1985, 1986), evokes sprouting of cholinergic septal and other afferents in the DG outer molecular layer. Our results showed a lesion-induced increase of bFGF immunoreactivity in the DG outer molecular layer ipsilateral to the lesion. The increase in bFGF immunoreactivity was expressed in three parameters: (1) an increase in the number of bFGF astrocytes, (2) an increase in the intensity of the bFGF immunoreactivity within astrocytes, and (3) an increase in the intensity of the bFGF immunoreactivity within the surrounding extracellular matrix. The time course and pattern of reorganization were similar to that of sprouting septal fibers found after such a lesion. Previously, it has been shown that bFGF acts on septal neurons to enhance their survival following axotomy (Anderson et al., 1987; Otto et al., 1989). Studies using in vitro preparations of septal neurons demonstrated that bFGF as well as NGF (Grothe et al., 1989; Knusel et al., 1990) can regulate the activity of the enzyme ChAT and/or stimulate neuritic outgrowth.

The fact that an increase in bFGF immunoreactivity was detected in the extracellular matrix of the DG of the lesion side suggests that bFGF is released from astrocytes and becomes available to other neural elements. Our observations that normal astrocytes show bFGF in the nucleus and perinuclear area and hyperreactive astrocytes around the lesion show bFGF in the cytoplasm suggest a possible translocation of bFGF toward secretion pathways. The mechanism of release of $b F G F$ from the cell is not well understood; however, it appears that bFGF reaches the cell surface by an active mechanism. A recent report indicates that one of the bFGF isoforms ( $18 \mathrm{kDa})$ is "exported" from the nucleus onto the cell surface by active transport (Florkiewicz et al., 1991). Heparan sulfate, a component of the 
extracellular matrix, increases the radius of diffusion and action of bFGF (Flaumenhaft et al., 1990), protects it from degradation (Gospadorowicz and Cheng, 1986), and potentiates its neurotrophic effect (Walicke, 1988b). The bFGF detected in our experiments probably represents bFGF in its active form since the monoclonal antibody to bFGF specifically recognizes the molecular structure of bFGF that is associated with its biological activity (Matsuzaki et al., 1989). Thus, the present results suggest that bFGF may be involved in the sprouting of septal and/ or other terminals within the dentate gyrus.

Our studies on entorhinal lesions in rodents may provide a model to understand the mechanisms responsible for changes in neuronal circuitry and plaque formation in AD. Geddes et al. $(1985,1986)$ have shown that axon sprouting occurs in the $\mathrm{DG}$ of $\mathrm{AD}$ brain, probably early in the disease. As the disease progresses, however, sprouting appears to become involved in senile plaque formation. It has been hypothesized that plaque formation may involve local increases in neurotrophic factors (Ramon y Cajal, 1928; Geddes et al., 1986), and recently bFGF has been identified in plaques of the DG (Gómez-Pinilla et al., 1990). According to the present study, it appears that the loss of entorhinal input that occurs in AD contributes to the stimulus leading to a local increase of bFGF in the dentate gyrus. This local increase of bFGF may become available to plaques as a result of its strong affinity for proteoglycans that are contained in plaques (Snow and Wright, 1989). Once within plaques, bFGF possibly attracts neurites, contributing to the process of plaque biogenesis.

Our results confirm previous observations that bFGF is able to ameliorate the death of axotomized cholinergic neurons (Anderson et al., 1988) and show that bFGF-responsive neurons retain NGFRs so these cells may be responsive to NGF. Thus, bFGF could play a critical role in neuronal survival by acting directly and/or indirectly on neurons. A possible indirect mechanism for bFGF action on neuronal survival is through the stimulation of astrocytic-made NGF, acting in turn on neuronal NGFRs. Astrocytes can synthesize NGF in vitro (Furukawa et al., 1986; Tarris et al., 1986), and bFGF has a recognized role as a mitogenic agent for astrocytes (Morrison and De Vellis, 1981; Pettmann et al., 1985). Thus, bFGF may work in an autocrine fashion, ensuring its own release by astrocytes, and stimulating the synthesis of NGF in astrocytes (Yoshida and Gage, 1991), and thus both newly synthesized bFGF and NGF would be made available for neuronal plasticity. Our results are consistent with recent studies (Cattaneo and McKay, 1990) indicating an interaction between bFGF and the expression of NGFR in cultured cells, and suggest that multiple growth factors may coordinate their actions to affect the physiology of particular neuronal populations.

In summary, our data on the widespread distribution of bFGF in the brain are in general agreement with the characteristic broad spectrum of its action. It appears that bFGF is induced after injury and in neurodegenerative disease, probably as part of a neurotrophic factor cascade in which astrocytes play a key role. As a result, bFGF can directly and/or indirectly stimulate neuronal repair and promote neuronal survival.

\section{References}

Abraham JA, Mergia A, Whang JL, Tumolo A, Friedman J, Hjerrild KA, Gospodarowicz D, Fiddes JC (1986) Nucleotide sequence of a bovine clone encoding the angiogenic protein, basic fibroblast growth factor. Science 233:545-548.
Anderson KJ, Dam D, Lee S, Cotman CW (1988) Basic fibroblast growth factor prevents death of lesioned cholinergic neurons in vivo. Nature 332:360-361.

Baird A, Walicke P (1989) Fibroblast growth factors. Br Med Bull 45: 438-452.

Cattaneo E, McKay R (1990) Proliferation and differentiation of neuronal stem cells regulated by nerve growth factor. Nature 347:762765.

Cotman CW, Anderson KJ (1989) Neural plasticity and regeneration. In: Basic neurochemistry, 4th ed (Siegel GJ, ed), pp 507-522. New York: Raven.

Cotman CW, Matthews DA, Taylor D, Lynch G (1973) Synaptic rearrangement in the dentate gyrus: histochemical evidence of adjustments after lesions in immature and adult rats. Proc Natl Acad Sci USA 70:3473-3477.

Daitz HM, Powell TPS (1954) Studies on the connexions of the fornix system. J Neurol Neurosurg Psychiatry 17:75-82.

Emoto M, Gonzalez AM, Walicke PA, Wada E, Simmons DM, Shimasaki S, Baird A (1989) Basic fibroblast growth factor (FGF) in the central nervous system: identification of specific loci of basic FGF expression in the rat brain. Growth Factors 2:21-29.

Esch F, Baird A, Ling N, Veno N, Hill F, Denoroy L, Klepper R, Gospodarowicz D, Bohlen P, Guilemin R (1985) Primary structure of bovine pituitary basic fibroblast growth factor (FGF) and comparison with the amino-terminal sequence of bovine brain acidic FGF. Proc Natl Acad Sci USA 85:6507-6511.

Ferrara N, Ousley F, Gospodarowicz D (1988) Bovine brain astrocytes express basic fibroblast growth factor, a neurotrophic angiogenic mitogen. Brain Res 462:223-232.

Finklestein SP, Apostolides PJ, Caday CG, Prosser J, Philips MF, Klagsbrun M (1988) Increased basic fibroblast growth factor (bFGF) immunoreactivity at the site of focal brain wounds. Brain Res 460:253259.

Flaumenhaft R, Moscatelli D, Rifkin DB (1990) Heparin and heparan sulfate increase the radius of diffusion and action of basic fibroblast growth factor. J Cell Biol 111:1651-1659.

Florkiewicz R, Baird A, Gonzalez AM (1991) Multiple forms of bFGF: differential nuclear and cell surface localization. Growth Factors 4: 265-275.

Folkman J, Klagsbrun M (1987) Angiogenic factors. Science 235:442446.

Furukawa S, Furukawa Y, Satoyoshi E, Hayashi K (1986) Synthesis and secretion of nerve growth factor by mouse astroglial cells in culture. Biochem Biophys Res Commun 136:57-63.

Gage FH, Wictorin K, Fischer W, Williams LR, Varon S, Björklund A (1986) Retrograde cell changes in medial septum and diagonal band following fimbria-fornix transection: quantitative temporal analysis. Neuroscience 19:241-255.

Gage FH, Olejniczak P, Armstrong D (1988) Astrocytes are important for sprouting in the septohippocampal circuit. Exp Neurol 102:2-13.

Geddes JW, Monaghan DT, Cotman CW, Lott IT, Kim RC, Chui HC (1985) Plasticity of hippocampal circuitry in Alzheimer's disease. Science 230:1179-1181.

Geddes JW, Anderson KJ, Cotman CW (1986) Scnilc plaques as aberrant sprout-stimulating structures. Exp Neurol 94:767-776.

Gómez-Pinilla F, Cotman CW, Nieto-Sampedro M (1987) NGF receptor immunoreactivity in rat brain: topographic distribution and response to entorhinal ablation. Neurosci Lett 82:260-266.

Gómez-Pinilla F, Cummings BJ, Cotman CW (1990) Induction of basic fibroblast growth factor in Alzheimer's disease pathology. Neuroreport 1:211-214.

Gospodarowicz D (1974) Localization of a fibroblast growth factor and its effect alone and with hydrocortisone on $3 \mathrm{t} 3$ cell growth. Nature 249:123-127.

Gospodarowicz D, Cheng J (1986) Heparin protects basic and acidic FGF from inactivation. J Cell Phyhsiol 128:475-484.

Gospodarowicz D, Cheng J, Lui G, Baird A, Bohlen P (1984) Isolation of brain fibroblast growth factor by heparin-sepharose affinity chromatography: identity with pituitary fibroblast growth factor. Proc Natl Acad Sci USA 81:6963-6967.

Grothe C, Otto D, Unsicker K (1989) Basic fibroblast growth factor promotes in vitro survival and cholinergic development of septal neurons: cmparisons with the effects of nerve growth factor. Neuroscience 31:649-661.

Hatten ME, Lynch M, Rydel RE, Sanchez J, Joseph-Silverstein J, Mos- 
catelli D, Rifkin DB (1988) In vitro neurite extension by granule neurons is dependent upon astroglial-derived fibroblast growth factor. Dev Biol 125:280-289.

Hoffman RS (1940) Growth-activating effect of extracts of adult and embryonic tissues of rat on fibroblast colonies in vitro. Growth 4: 361-376.

Inamura T, Tokita Y, Mitsui Y (1988) Purification of basic FGF receptors from rat brain. Biochem Biophys Res Commun 155:583590.

Isacchi A, Bergonzoni L, Sarmientos P (1990) Complete sequence of a human receptor for acidic and basic fibroblast growth factors. Nucleic Acids Res 18:2616.

Knusel B, Michel PP, Schwaber JS, Hefti F (1990) Selective and nonselective stimulation of central cholinergic and dopaminergic development in vitro by nerve growth factor, basic fibroblast growth factor, epidermal growth factor, insulin and the insulin-like growth factor I and II. J Neurosci 10:558-570.

Kromer LF (1987) Nerve growth factor treatment after brain injury prevents neuronal death. Science 235:214-216.

Lee PL, Johnson DE, Cousen LS, Fried VA, Williams LT (1989) Purification and complementary DNA cloning of a receptor for basic fibroblast growth factor. Science 245:57-60.

Matsuzaki K, Yoshitake Y, Matuo Y, Sasaki H, Nishikawa K (1989) Monoclonal antibodies against heparin-binding growth factor $1 \mathrm{I} / \mathrm{ba}-$ sic fibroblast growth factor that block its biological activity: invalidity of the antibodies for tumor angiogenesis. Proc Natl Acad Sci USA 86:9911-9915.

McGeer PL, Akiyama H, Itagaki S, McGeer EG (1989) Immune system response in Alzheimer's disease. Can J Neurol Sci 16:516-527.

Montero CN, Hefti F (1988) Rescue of lesioned septal cholinergic neurons by nerve growth factor: specificity and requirement for chronic treatment. J Neurosci 8:2986-2999.

Morrison RS, De Vellis J (1981) Growth of purified astrocytes in a chemically defined medium. Proc Natl Acad Sci USA 78:7205-7209.

Nieto-Sampedro M, Lewis ER, Cotman CW, Manthorpe M, Skaper SD, Barbin G, Longo FM, Varon S (1982) Brain injury causes a timedependent increase in neurotrophic activity at the lesion site. Science 221:860-861.

Otto D, Frotscher M, Unsicker K (1989) Basic fibroblast growth factor and nerve growth factor administered in Gelfoam rescue medial septal neurons after timbria fornix transection. J Neurosci Res 22:83-91.

Pettmann B, Weibel M, Sensenbrenner M, Labourdette G (1985) Purification of two astroglial growth factors from bovine brain. FEBS Lett 189:102-108.

Pettmann B, Labourdette G, Weibel M, Sensenbrenner M (1986) The brain fibroblast growth factor (FGF) is localized in neurons. Neurosci Lett 68:175-180.

Ramon y Cajal S (1928) Study of regenerative processes of the ce- rebrum. Degeneration and regeneration of the nervous system, Vol II (May RM, ed) pp 734-762. London: Hafner.

Reid HH, Wilks AF, Bernard O (1990) Two forms of the basic fibroblast growth factor receptor-like mRNA are expressed in the developing mouse brain. Proc Natl Acad Sci USA 87:1596-1600.

Rose G, Lynch G, Cotman CW (1976) Hypertrophy and redistribution of astrocytes in the deafferented dentate gyrus. Brain Res Bull 1:8792.

Schweigerer L, Neufeld G, Friedman J, Abraham JA, Fiddes JC, Gospodarowicz D (1987) Capillary endothelial cells express basic fibroblast growth factor, a mitogen that promotes their own growth. Nature 325:257-259.

Sievers J, Hausmann B, Unsicker K, Berry M (1987) Fibroblast growth factors promote the survival of adult rat retinal ganglion cells after transection of the optic nerve. Neurosci Lett 76:157-162.

Smolen AJ (1990) Image analytic techniques for quantitation of immunohistochemical staining in the nervous system. Methods Neurosci 3:208-229.

Snow AD, Wright TN (1989) Proteoglycans in the pathogenesis of Alzheimer's disease and other amyloidoses. Neurobiol Aging 10:481497.

Sofroniew MV, Pearson RCA, Powell TPS (1987) The cholinergic nuclei of the basal forebrain of the rat: normal structure, development and experimentally induced degeneration. Brain Res 411:310-331.

Stopa EG, Gonzalez A, Chorsky R, Corona R, Alvarez J, Bird ED, Baird A (1990) Basic fibroblast growth factor in Alzheimer's disease. Biochem Biophys Res Commun 171:690-696.

Tarris RH, Weichsel JR, Fisher DA (1986) Synthesis and secretion of a nerve growth-stimulating factor by neonatal mouse astrocyte cells in vitro. Pediatric Res 20:367-372.

Trowell OA, Willmer EN (1939) Studies on growth of tissues in vitro; effects of some tissue extracts on growth of periosteal fibroblasts. J Exp Biol 16:60-70.

Vijayan VK, Geddes JW, Anderson KJ, Chang-Chui H, Cotman CW (1991) Astrocyte hypertrophy in the Alzheimer's disease hippocampal formation. Exp Neurol 112:72-78.

Walicke PA (1988a) Interactions between basic fibroblast growth factor (FGF) and glycosoaminoglycans in promoting neurite outgrowth. Exp Neurol 102:144-148.

Walicke PA (1988b) Basic and acidic fibroblast growth factors have trophic effects on neurons from multiple CNS regions. J Neurosci 8: 2618-2627.

Walicke PA, Baird A (1988) Trophic effects of fibroblast growth factor or neural tissue. Prog Brain Res 78:333-338.

Yoshida K, Gage F (1991) Fibroblast growth factors stimulate nerve growth factor synthesis and secretion by astrocytes. Brain Res 538: 118-126. 